\title{
Optimal Kernel Choice for Domain Adaption Learning
}

\author{
Le Dong ${ }^{\mathrm{a}, *}$, Ning Feng ${ }^{\mathrm{a}}$, Pinjie Quan ${ }^{\mathrm{a}}$, Gaipeng Konga ${ }^{\mathrm{a}}$, Xiuyuan $\mathrm{Chen}^{\mathrm{a}}$, \\ Qianni Zhang ${ }^{\mathrm{b}}$ \\ ${ }^{a}$ School of Computer Science and Engineering, University of Electronic Science and \\ Technology of China (UESTC),2006 Xiyuan Avenue, Gaoxin West Zone, Chengdu, \\ Sichuan, 611731, China \\ ${ }^{b}$ School of Electronic Engineering and Computer Science, Queen Mary, University of \\ London
}

\begin{abstract}
In this paper, a kernel choice method is proposed for domain adaption, referred to as Optimal Kernel Choice Domain Adaption (OKCDA). It learns a robust classier and parameters associate with Multiple Kernel Learning side by side. Domain adaption kernel-based learning strategy has shown outstanding performance. It embeds two domains of different distributions, namely, the auxiliary and the target domains, into Hilbert Space, and exploits the labeled data from the source domain to train a robust kernel-based SVM classier for the target domain. We reduce the distributions mismatch by setting up a test statistic between the two domains based on the Maximum Mean Discrepancy (MMD) algorithm and minimize the Type II error, given an upper bound on error I. Simultaneously, we minimize the structural risk functional. In order to highlight the advantages of the proposed method, we tackle a text classication problem on 20 Newsgroups dataset and Email Spam dataset. The results demonstrate that our method exhibits outstanding performance.
\end{abstract}

Keywords: Optimal Kernel, Domain Adaption, Cross-domain, Test Statistic, Kernel Choice

\footnotetext{
${ }^{*}$ Corresponding author. Tel.:+86 13981763623; Fax: +86-28-61831655.

Email address: ledong@uestc.edu.cn (Le Dong)
} 


\section{Introduction}

Conventional machine learning methods universally assume that the training data and the test data come from the same distribution. Unfortunately for many applications, it is difficult to obtain enough labeled data for training classifiers.

5 Recently, many researchers have been focusing on cross-domain adaption which aims at solving a learning problem in the target domain by utilizing training data in the source domain, while these two domains may have different distributions [1, 2]. In practice, the domain adaptive learning strategy has been successfully applied to real-time applications, such as multi-task clustering [3]

${ }_{10}$,WiFi localization [4, action recognition [5], sentiment classification [6], visual event recognition [7, 8, object detection [9, 10, and visual concept classification [11, 12, 13]. However, compared with non-learning methods [14, 15], adaptive learning has more extensive applications.

To take the advantage of all labeled patterns for both auxiliary and tar15 get domains, Daume [16] proposes a Feature Replication method to augment features for cross-domain learning. The augmented features are then used to construct a kernel function for Support Vector Machine training. Yang et al. [12] propose Adaptive SVM for visual concept classification, in which the new classifier $f^{T}(x)$ is adapted from an existing classifier $f^{A}(x)$ trained from the neighbors from the target domain to define a weight for each auxiliary pattern, and then the SVM classifier is trained with the re-weighted auxiliary patterns. More recently, Jiang et al. [11 proposes a method of mining the relationship among different visual concepts for video concept detection. They first build a semantic graph which can be adapted in an online fashion to fit the new knowledge mined from the test data. However, these methods do not utilize unlabeled patterns from the target domain. Such unlabeled patterns can also be used to improve the classification performance.

When there are only a few or even no labeled patterns available in the target domain, the auxiliary patterns or the unlabeled target patterns can be used to 
train the target classifier. Several cross-domain learning methods are proposed to cope with the inconsistency of data distributions. These methods re-weighted the training samples from the source domain by using unlabeled data from the target domain so that the statistics of samples from both domains are matched.

35 Duan et al. [17, 19] propose a cross-domain kernel learning framework, which learns a kernel function and classifier by minimizing both the structural risk functional and the distribution mismatch between the labeled and unlabeled samples from the auxiliary and target domains. This framework employs a domain similarity measure based on MMD. More recently, Duan et al. [7] develop a cross-domain learning method, referred to as Adaptive Multiple Kernel Learning (A-MKL) that has been successfully used in visual event recognition.

A common insight is that most of those domain adaption learning methods are either variants of SVM or other kernel methods, which map auxiliary data and target data into a feature space for obtaining a robust SVM-based classifier, 45 and simultaneously, minimize the mismatch between two different distribution domains. The performance of a classifier strongly depends on the choice of the kernels. Lanckriet et al. [18] develop a nonparametric kernel matrix, which involves joint optimization of the coefficients in a conic combination of kernel matrices. One problem is that its time complexity is too high to be applied to real applications. In recent years, many effective methods [17, 19, 20, 21, 22] have been developed to combine multiple kernels instead of directly learning the kernel matrix, in which the kernel function is a linear combination of based kernel functions. However, all those methods suppose that both test and training data are drawn from the same distribution. Consequently, naked multiple kernel learning cannot directly solve the problem of cross-domain learning. Because the coefficients of combination kernel are parameterized, the training data from source domain may degrade the performance of the model in the target domain.

In this paper, we propose a new method on kernel choice for cross-domain learning, which explicitly minimizes the loss due to the bias between the data distributions of the auxiliary and target domains, as well as the cost function of structural risk for all labeled patterns. Type I error is the probability of 
wrongly rejecting null hypothesis when the auxiliary distribution and the target distribution are drawn from the same distribution. Type II error is the probability of wrongly accepting null hypothesis when the auxiliary and the target distributions are different. Given an upper bound on Type I error, our kernel choice minimizes Type II error. The main contribution of this paper is that multiple base kernels are weighted to minimize the loss on the labeled examples and the bias between the data distributions in the two domains. Meanwhile, we minimize the bias between the source domain and the target domain by minimizing the Type II error. While multi-kernel method has been widely discussed [23, 24, 20] and used [21], our work demonstrates that the kernel choice is pivotal to cross-domain learning.

The rest of paper is organized as follows: We briefly review the related works in Section 2. Section 3 introduces kernel choice for domain adaption learning. We experimentally compare the proposed method with other crossdomain learning methods on the 20 Newsgroups dataset and Email Spam dataset for text classification in Section 4. Finally, conclusion is made in Section 5.

\section{Brief Review of Related Work}

Let us denote the dataset of labeled and unlabeled patterns from the target domain as $D_{l}^{T}=\left.\left(x_{i}^{T}, y_{i}^{T}\right)\right|_{i=1} ^{n_{l}}$ and $D_{u}^{T}=\left.\left(x_{i}^{T}, y_{i}^{T}\right)\right|_{i=n_{l}+1} ^{n_{l}+n_{u}}$, respectively, where $y_{i}^{T}$ is the label of $x_{i}^{T}$, labeled patterns are numbered 1 to $n_{l}$, unlabeled patterns are numbered $n_{l}+1$ to $n_{l}+n_{u}$. We define $D^{T}=D_{l}^{T} \bigcup D_{u}^{T}$ as the dataset from the target domain with the size $n_{t}=n_{l}+n_{u}$ under the marginal data distribution $\rho$, and $D^{A}=\left.\left(x_{i}^{A}, y_{i}^{A}\right)\right|_{i=1} ^{n_{A}}$ as the dataset from the source domain under the marginal data distribution $\vartheta$. We represent the labeled training dataset as $D=\left.\left(x_{i}, y_{i}\right)\right|_{i=1} ^{n}$, where $\mathrm{n}$ is the total number of labeled patterns. The labeled training data can be from the target domain $\left(D=D_{l}^{T}\right)$ or from both domains $\left(D=D_{l}^{T} \cup D^{A}\right)$. 


\subsection{Minimize Bias of Distribution Using Test Statistic}

It is important to reduce the mismatch between the source domain and the target domain distributions, and many methods have been proposed to address this work. A classic criteria is Kullback Leibler divergence 25]. However, most of them are parametric and need to estimate an intermediate density. To steer clear of fussy measure, Borgwardt et al. [26] present a novel non-parametric statistical method, namely, Maximum Mean Discrepancy, which is based on Reproducing Kernel Hilbert Space [27.

$$
\begin{aligned}
& M M D\left(D^{A}, D^{T}\right)=\sup _{\|f\|_{H} \leq 1}\left(E_{x^{A} \sim Q}\left[f\left(x^{A}\right)\right]-E_{x^{T} \sim P}\left[f\left(x^{T}\right)\right]\right) \\
& =\sup _{\|f\|_{H} \leq 1}\left\langle f,\left(E_{x^{A} \sim Q}\left[f\left(x^{A}\right)\right]-E_{x^{T} \sim P}\left[f\left(x^{T}\right)\right]\right)\right. \\
& =\left\|E_{x^{A} \sim Q}\left[f\left(x^{A}\right)\right]-E_{x^{T} \sim P}\left[f\left(x^{T}\right)\right]\right\|_{H},
\end{aligned}
$$

where $E_{x \sim \mu}[\cdot]$ denotes the expectation operator under the samples distribution $\mu$ and $f(x)$ is any function in $H$. The second equality holds as $f(x)=\langle f, \phi(x)\rangle_{H}$ by the property of RKHS, where $\phi(x)$ is the nonlinear feature mapping of the kernel $k$. Note that the inner product of $\phi\left(x_{i}\right)$ and $\phi\left(x_{j}\right)$ equals to the kernel function $k(\cdot, \cdot)$ on $x_{i}$ and $x_{j}$, namely, $k\left(x_{i}, x_{j}\right)=\phi\left(x_{i}\right) \phi\left(x_{j}\right)$. An expression for the squared MMD is

$$
\begin{aligned}
& \eta_{k}\left(D^{A}, D^{T}\right)=\left\|\phi\left(D^{A}\right)-\phi\left(D^{T}\right)\right\|_{H}^{2} \\
& =E_{x x^{\prime}} k\left(x, x^{\prime}\right)+E_{y y^{\prime}} k\left(y, y^{\prime}\right)-2 E_{y x^{\prime}} k\left(y, x^{\prime}\right),
\end{aligned}
$$

90

where $x, x^{\prime} \sim^{i . i . d} p$ and $y, y^{\prime} \sim^{i . i . d} q$. By introducing $h_{k}\left(x, x^{\prime}, y, y^{\prime}\right)=k\left(x, x^{\prime}\right)+$ $k\left(y, y^{\prime}\right)-k\left(y, x^{\prime}\right)-k\left(x, y^{\prime}\right)$, Eq. 22 can be rewritten as $\eta_{k}=\mathbf{E}_{x x^{\prime} y y^{\prime}} h_{k}\left(x, x^{\prime}, y, y^{\prime}\right)$. By introducing $h_{k}\left(x, x^{\prime}, y, y^{\prime}\right)=k\left(x, x^{\prime}\right)+k\left(y, y^{\prime}\right)-k\left(y, x^{\prime}\right)-k\left(x, y^{\prime}\right)$, Eq. 2 can be rewritten as $\eta_{k}=\mathbf{E}_{x x^{\prime} y y^{\prime}} h_{k}\left(x, x^{\prime}, y, y^{\prime}\right)$. In brief, the key point of MMD is that the distance between distributions of two domains is equivalent to the 95 distance between the means of the two domains mapped into a RKHS [4]. Huang et al. 28] develop a two-step method. The first step is to diminish the mismatch of means of different distributions in RKHS by reweighting the examples using 
square MMD. The seconed step is to learn a decision function that separates patterns from two opposite classes. One difficulty is that the performance of MMD strongly depends on the choice of kernel. Meanwhile, these methods do not ensure that the chosen kernel is optimal. Inspired by [29], we review the problem of bias between the source domain and the target domain as a twosample test problem, which addresses the question of whether two independent samples are drawn from the same distribution. Consequently, given two example distributions: $q$ from source (auxiliary) domain and $p$ from target domain, we can set up a two-sample test which measures the similarity or bias between the source domain and the target domain.

We select some kernels for hypothesis testing from a particular family $\boldsymbol{K}$ of kernels, assuming kernel $k\left(x_{i}, x_{j}\right)$ is a linear combination of a set of base kernels

$$
k_{\mathbf{d}}=\sum_{m=1}^{M} d_{m} k_{m},
$$

where $d_{m}>0$ is a set of positive coefficients, $\sum_{m=1}^{M} d_{m}=D>0$. The squared MMD becomes

$$
\eta_{m}\left(D^{A}, D^{T}\right)=\left\|\phi\left(D^{A}\right)-\phi\left(D^{A}\right)\right\|_{\digamma}^{2}=\sum_{l=1}^{M} d_{l} \eta_{l}\left(D^{A}, D^{T}\right) .
$$

Here, it is denoted that $d=\left\{d_{1}, d_{2}, \ldots, d_{M}\right\}^{T} \in R^{M \times 1}, \eta=\left\{\eta_{1}, \eta_{2}, \ldots, \eta_{M}\right\} \in$ $R^{M \times 1}$. Eq. (5) can be written as $\eta_{m}\left(D^{A}, D^{T}\right)=d^{T} \eta$. $\eta_{m}$ is the average of independent random variables, and its asymptotic distribution is given by the central limit theorem. Now we set up the construction of a hypothesis test and define $\Phi$ as the Cumulative Distribution Function (CDF) of a standard normal random variable $N(0,1)$, where $\Phi^{-1}$ is the inverse CDF. A test of asymptotic level $\alpha$ using the statistics will have a threshold $t$ as in [14]. To obtain an estimate of the variance based on the samples, we use an expression derived from the U-statistic. The population variance can be written as

$$
\sigma^{2}=E_{x x^{\prime} y y^{\prime}} h_{k}^{2}\left(x, x^{\prime}, y, y^{\prime}\right)-\left[E_{x x^{\prime} y y^{\prime}} h_{k}\left(x, x^{\prime}, y, y^{\prime}\right)\right]^{2} .
$$

The choice of kernel will affect both the test statistic and the asymptotic variance. Type II error occurs when the random variable $\eta_{k}$ falls below the threshold 
$t$. The asymptotic probability of a Type II error is therefore expressed as:

$$
P\left(\eta_{m}<t\right)=\Phi\left(\Phi^{-1}(1-\vartheta)-\eta_{k}\left(D^{A}, D^{T}\right) \sqrt{n} / \sqrt{2} \sigma^{k}\right)
$$

where $\Phi$ is a monotonic function, $n$ is the number of all patters and $t$ is the threshold of the test statistic and is set to $\sqrt{2} n^{-1 / 2} \sigma_{k} \Phi^{-1}(1-\vartheta)$. Obviously, the Type II probability will decrease as the ratio $\eta_{m}\left(D^{A}, D^{T}\right) \sigma_{k}^{-1}$ increases.

\subsection{Borrow Knowledge From Ready-Made Classifier}

Instead of directly utilizing the source domain data to learn a classifier, many researchers consider learning a final classifier from a pre-learned classifier trained by an source domain [17. Yang et al. [12] develop Adaptive SVM, in learning methods [31, 32 are proposed. Pan et al. 4 demonstrate a Maximum Mean Discrepancy Embedding, which minimizes the square of the Maximum Mean Discrepancy criterion, and then applies the learned kernel matrix to train SVM classifier for WiFi localization and text categorization. Different from are better in extending the previous work on optimal kernel choice to two-sample tests. The proposed approaches are described in full details in section 3 . 


\section{Optimal Kernel Choice for Domain Adaption Learning}

Similar to previous methods, we assume the kernel function is a linear combination of a set of base kernels. Our goal is to learn a function of the form $f(x)=w \varnothing(x)+b$ with multiple kernels $k\left(x_{i}, x_{j}\right)=\sum_{i=1}^{d} \beta_{i} k\left(x_{i}, x_{j}\right)$ representing the inner product in a feature space parameterized by $\beta$. At the same time, we minimize the bias of different distributions between the source domain and the target domain. The definition of an object function for domain adaption learning can be formulated as

$$
\arg \min _{d \in D} \Phi\left(P\left(\eta_{k}<t\right)\right)+\lambda \Upsilon(d)
$$

where

$$
\begin{aligned}
& \Upsilon(d)=\min _{w, \xi, b}\|w\|^{2} / 2+C \sum_{i=1}^{l} \xi_{i}+\Omega(d) \\
& \text { s.t. } \quad 1-\xi_{i} \leq y_{i}\left(\sum_{i}^{n} w_{i} \phi\left(x_{i}\right)+b\right), 0 \geq \xi, 0 \leq d . \\
& \text { where } \quad \phi\left(x_{i}\right) \phi\left(x_{j}\right)=\sum_{i=1}^{M} d_{i} \phi_{i}\left(x_{i}\right) \phi_{i}\left(x_{j}\right)
\end{aligned}
$$

Note that $\Phi(\cdot)$ is a monotonic increasing function, and $\Omega(d)$ is constraint function with $d . n$ is the total number of training samples. $\Upsilon(d)$ is the regularizer function and can be any differentiable function of $d$ with continuous dervative. $\lambda$ is a tradeoff parameter. $\mathrm{C}>0$ is regularization parameter where the objective term is near to the standard $C$-SVM objective term. Given the misclassification $\mathrm{C}$, the aim is to maximise the margin while minimizing the hinge loss on the labeled data between the auxiliary and the target domains. The only addition is an regularisation on the weights $d$ associated with multiple kernels. Recall in [29] it is shown that minimizing the Type II error, $P\left(\eta_{k}<t\right)$, is equal to minimizing $d^{\prime} Q d$, where $Q$ is the covariance matrix $\operatorname{cov}(h)$. The models [29] are referred to full details. Let us define

$$
J(d)=d^{\prime} Q d / 2+\lambda \Omega(d) .
$$


then, the optimization problem can be rewritten as

$$
\begin{aligned}
& \min _{d \in D} T(d)=\min _{w, b, \xi} J(d)+\lambda\left(w^{\prime} w / 2+C \sum_{i=1}^{l} \xi_{i}\right) \\
& \text { s.t. } \quad 1-\xi_{i} \leq y_{i}\left(\sum_{i}^{d} w_{i} \varnothing\left(x_{i}\right)+b\right) \\
& \quad 0 \leq \xi, 0 \leq d .
\end{aligned}
$$

However, the objective function term in the brackets and the constraints are the standard $C$-SVM object. $\lambda$ is set to $0.1,0.2,0.5,1,2,5,10,20,50$. It is straightforward to derive the corresponding dual problem

$$
\begin{gathered}
W(d)=\max _{\alpha} \quad \mathbf{1}^{T} \alpha-1 / 2 \alpha_{d}^{T} \alpha+J(d) \\
\mathbf{1}^{T} Y \alpha=0, \quad 0 \leq \alpha \leq C,
\end{gathered}
$$

where $K_{d}$ is the kernel matrix for a given $d, Y$ is a diagonal matrix with the labels on the diagonal. In this paper, we utilize project gradient descent in the outer loop to obtain $d$. According to [22, $W$ can be differentiated with respect to $d$ as if $\alpha^{*}$ does not depend on $d$. We therefore get

$$
\begin{aligned}
& \partial T / \partial d=\partial W / \partial d=\partial J / \partial d-\alpha^{*} T Y\left(\partial K_{d} / \partial d\right) Y \alpha^{*} / 2 \\
& \partial^{2} T / \partial d^{2}=\partial^{2} W / \partial d^{2}=\partial^{2} J / \partial d^{2} .
\end{aligned}
$$

In our learning method, we employ the reduced gradient descent procedure proposed by iteratively updating the linear combination coefficient $d$ and the dual variable $\alpha$. There are two stages in this process:

The first stage: $d$ is fixed, thus $\Omega(d)$ is a constant. We solve Eq.14 to obtain the dual variable $\alpha$.

The second stage: Based on the first stage, $\alpha$ is fixed. Use the projected gradient descent process method to obtain d. With respect to $d$, the objective function $M(d)$ can be rewritten as

$$
M(d)=d^{\prime} Q d / 2+\mathbf{1}^{\prime} \alpha-\alpha^{T} Y K_{d} Y \alpha / 2+J(d) .
$$


We adopt the second-order gradient descent method to update the linear combination coefficient $d$ at iteration $k+1$ by

$$
d^{k+1}=d^{k}-\varepsilon^{k} \nabla^{2} G,
$$

where $\varepsilon_{t}$ is the learning rate which can be computed by using a line search method, in which $\nabla^{2} G$ is the updating direction. It is worthwhile to note that $Q$ is not a full rank. To avoid numerical instability, we define $Q=Q+\varsigma \mathbf{E}$, where $\varsigma$ is set to $10^{-6}$ in the experiment.

\section{Experiment}

In this section, we'll evaluate the effectiveness of our approach. We compare our kernel choice approach for domain adaption, with the baseline SVM, and some state-of-the-art domain adaptation learning methods, such as Feature Replication [16], Adaptive SVM (A-SVM) 12], CDSVM [13], MKL and DMKLDF [17]. In our experiment, we focus on challenging text classification problems on 20 Newsgroups dataset 1 and Email Spam dataset.

\subsection{Datasets Description}

The 20 Newsgroups dataset is a collection of approximately 20000 newsgroup documents, partitioned (nearly) evenly across six main categories and 20 different newsgroups, each corresponding to a different topic. Some of the newsgroups are very closely related to each other (e.g. comp.sys.ibm.pc.hardware vs. comp.sys.mac.hardware), while some others are highly unrelated (e.g misc.forsale vs. soc.religion.christian). The 20 newsgroups collection is a popular dataset for experiments in text applications of machine learning techniques.

In our experiment, we follow experiment setting of [7]. The four largest main categories are chosen for evaluation. Specifically, for each main category, the subcategory is chosen as the source domain. Table 1 provides the detailed

\footnotetext{
${ }^{1}$ Available at http://qwone.com/ jason/20Newsgroups/
} 


\begin{tabular}{|c|c|c|}
\hline Setting & Source Domain & Target Domain \\
\hline rec vs. sci & rec.sport.hockey and sci.crypt & rec.motocycles and sci.med \\
\hline rec vs. talk & rec.sport.hockey and talk.politics.mideast & rec.motocycles and talk.politics.guns \\
\hline
\end{tabular}

Table 1: Description of 20 Newsgroups Dataset

information of selected two settings. To build the training dataset, we also use all labeled samples from the source domain, and at the same time randomly choose $m$ positive and $m$ negative samples from the target domain. In the experiment, $m$ is set to $0,1,3,5,7$ and 10 . There are three email subsets (denoted by User1, User2, and User3, respectively) annotated by three different users in the email spam dataset. The task is to classify spam and non-spam emails.

\begin{tabular}{|c|c|c|}
\hline Setting & Source Domain & Target Domain \\
\hline User0 vs. User1 & User0 & User1 \\
\hline User1 vs. User2 & User1 & User2 \\
\hline User2 vs. User0 & User2 & User0 \\
\hline
\end{tabular}

Table 2: Description of Email Spam Dataset

Since the spam and non-spam emails in the subsets have been differentiated by different users, the data distributions of the three subsets are related but different. Each subset has 2500 emails, in which half of the emails are nonspam (labeled as 1) and the other half of them are spam (labeled as -1). In this dataset, we consider three settings as in Table 2. For each setting, the training dataset contains all labeled samples from the source domain as well as the labeled samples from the target domain, in which five positive and five negative samples are randomly chosen, and the remaining samples in the target domain are used as unlabeled training data and test data as well. We randomly sample the training data from the target domain for five times and report the means and the standard deviations of all methods. Again, the word-frequency feature is used to represent each document. 


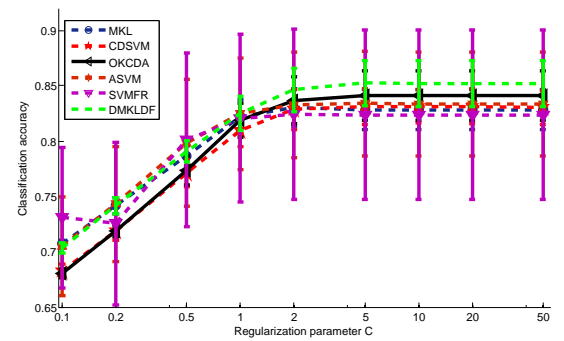

(a) rec vs. talk $\mathrm{m}=7(0.841 \pm 0.022)$

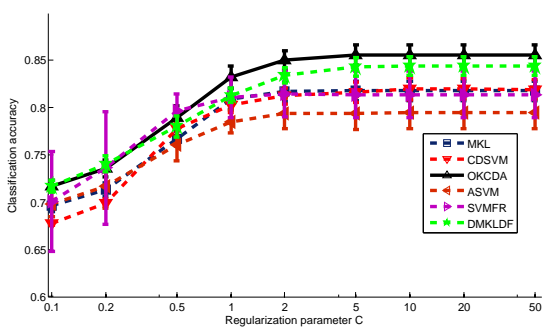

(c) rec vs. sci $\mathrm{m}=7(0.856 \pm 0.011)$

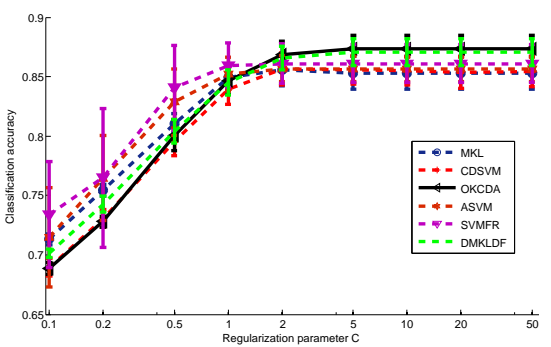

(b) rec vs. talk $\mathrm{m}=10(0.883 \pm 0.021)$

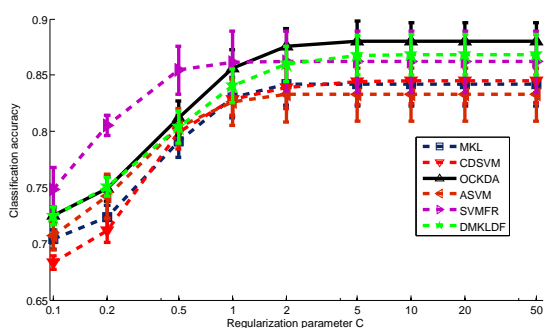

(d) rec vs. sci $\mathrm{m}=10(0.880 \pm 0.017)$

Figure 1: Performance comparisons of OKCDA with other methods in terms of the means and standard deviations of classification accuracies on the 20 Newsgroups dataset with different regularization parameters $C \in\{0.1,0.2,0.5,1,2,5,10,20,50\}$. We set $m=7$ or $m=10$.

\subsection{Experiment Setup}

Our base kernels are predetermined for all methods. Specifically, the following kernels have been used: Gaussian kernel(i.e., $\left.k\left(x_{i}, x_{j}\right)=\exp \left(-\gamma\left\|x_{i}-x_{j}\right\|\right)\right)$, Linear kernel (i.e., $\left.k\left(x_{i}, x_{j}\right)=x_{i} \cdot x_{j}\right)$ and Polynomial kernel (i.e., $k\left(x_{i}, x_{j}\right)=$ $\left.\left(x_{i} \cdot x_{j}+1\right)^{\gamma}\right)$, where the kernel parameter $\gamma$ is set as the default value 0.0005 . We use 10 kernel parameters $1.5^{\xi+1} \gamma, \xi \in\{-2.5,-2, \cdots, 2,2.5\}$. Motivated by [33], the regularization term of $J(\mathbf{d})$ is used, which is differentiable and continuous. $l_{1}$ regularization with $J(\mathbf{d})=\mathbf{d}$ or variations could be used for learning sparse solutions. Alternatively, $l_{2}$ regularization of the form $J(\mathbf{d})=(\mathbf{d}-\mu)^{\prime} \Sigma^{-1}(\mathbf{d}-\mu)$ can be used only when a small number of relevant kernels are present or if prior knowledge in the form $\mu$ and $\Sigma$ is available.

For performance evaluation, we use non-interpolated Average Precision (AP), 


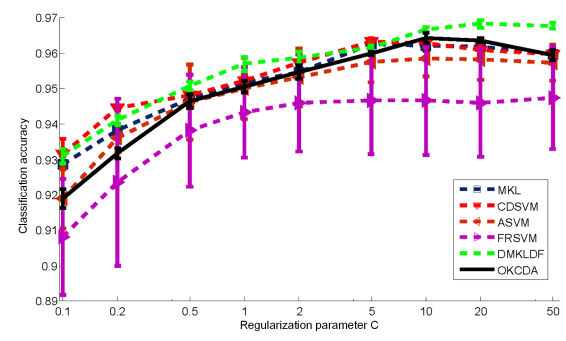

(a) User00 vs. User01 $(\mathrm{m}=7)$

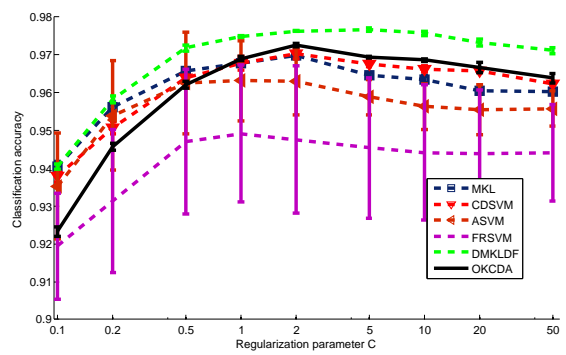

(c) User01 vs. User02 $(\mathrm{m}=7)$

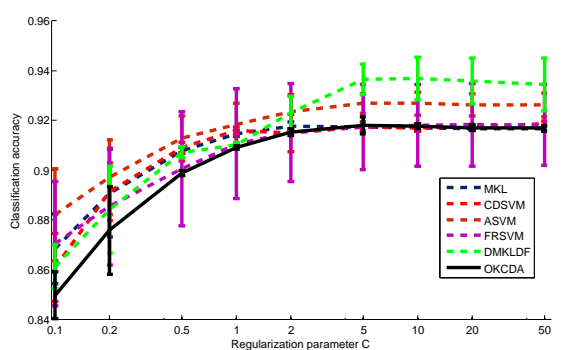

(e) User02 vs. User00 $(\mathrm{m}=7)$

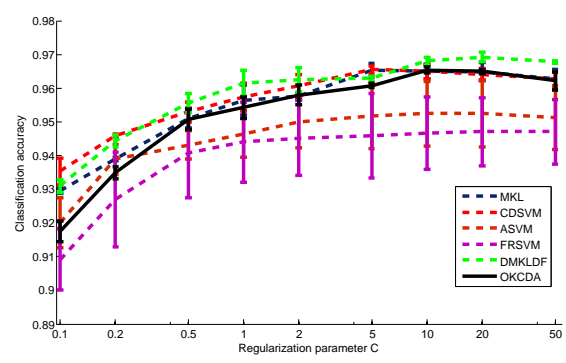

(b) User00 vs. User01 $(\mathrm{m}=15)$

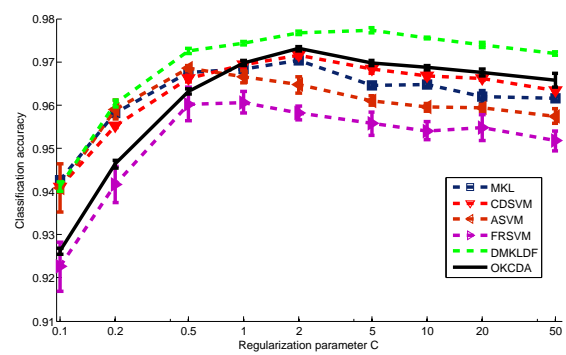

(d) User01 vs. User02 $(\mathrm{m}=15)$

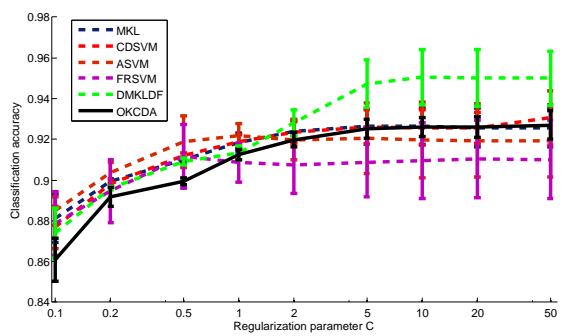

(f) User02 vs. User00 $(\mathrm{m}=15)$

Figure 2: Performance comparisons of OKCDA with other methods in terms of the means and standard deviations of classification accuracies on the Email Spam dataset with different regularization parameters $C \in\{0.1,0.2,0.5,1,2,5,10,20,50\}$. We set $m=7$ or $m=10$. 
which has been used as the official performance metric in TRECVID since 2001. AP is related to multipoint Average Precision value of a precision-recall curve and incorporates the effect of recall when it is computed over the entire classification results [34. Thanks to previous work [7, some Matlab code has been referenced for this purpose in our experiment. Table 3 shows the classification accuracies and standard deviations of classification accuracies of different methods on the real dataset. We obtain that the performance of our model improves obviously with the increasing of $\mathrm{m}$, and achieves the best result when $\mathrm{m}$ is set to 10 .

Table 4 presents the best among all the results obtained by using different regularization parameters $C \in\{0.1,0.2,0.5,1,2,5,10,50\}$.

\subsection{Results of 20 Newsgroups Dataset}

In our experiment, we also compare our proposed method with the competitive methods on the classification performance, including MKL, ASVM, CDSVM, SVMFR and DMKLDF. Different regularization parameters are used. Fig. 1 shows the variation in classification accuracies with varying $C$ over a range $[0.1,0.2,0.5,1,2,5,10,50]$. Note that the x-axis in Fig. 1 1 are in logarithmic scale. The results of all methods are obtained by using $\mathrm{m}$ positive and $\mathrm{m}$ negative training samples from the target domain, as well as the training data from the source domain, where $\mathrm{m}=7$ and 10 for the 20 Newsgroups dataset. We have the following observations:

From the Fig. 1] we observe that when $\mathrm{C}$ becomes larger, all methods tend to have better performance. However, our method OKCDA outperforms most other methods, only except DMKLDF in terms of mean classification accuracies. Fig. 1(a) shows that SVMFR has the largest standard deviations of classification accuracies.

- We observe from Fig. 1(b), Fig. 1(c) and Fig. 1(d) that standard deviations of classification accuracies of SVMFR severely changes as $C$ from 0.1 to 2. We also observe that the growth pattern of classification accuracy for 
(a) rec vs. sci

\begin{tabular}{|c|c|c|c|c|c|c|}
\hline $\mathrm{m}$ & ASVM & CDSVM & MKL & SVMFR & DMKLDF & OKCDA \\
\hline 0 & & & $0.702 \pm 0.000$ & $0.668 \pm 0.000$ & $\mathbf{0 . 7 2 5} \pm \mathbf{0 . 0 0 0}$ & $0.713 \pm 0.000$ \\
\hline 1 & $0.687 \pm 0.043$ & $0.723 \pm 0.020$ & $0.742 \pm 0.022$ & $0.674 \pm 0.043$ & $0.750 \pm 0.021$ & $\mathbf{0 . 7 6 4} \pm \mathbf{0 . 0 2 2}$ \\
\hline 3 & $0.728 \pm 0.039$ & $0.758 \pm 0.024$ & $0.755 \pm 0.021$ & $0.719 \pm 0.056$ & $0.786 \pm 0.023$ & $\mathbf{0 . 7 9 9} \pm \mathbf{0 . 0 2 6}$ \\
\hline 5 & $0.770 \pm 0.028$ & $0.799 \pm 0.011$ & $0.795 \pm 0.013$ & $0.774 \pm 0.034$ & $0.822 \pm 0.021$ & $\mathbf{0 . 8 3 6} \pm \mathbf{0 . 0 1 3}$ \\
\hline 7 & $0.795 \pm 0.017$ & $0.820 \pm 0.011$ & $0.818 \pm 0.010$ & $0.814 \pm 0.024$ & $0.844 \pm 0.010$ & $\mathbf{0 . 8 5 6} \pm \mathbf{0 . 0 1 1}$ \\
\hline 10 & $0.833 \pm 0.023$ & $0.845 \pm 0.020$ & $0.842 \pm 0.018$ & $0.862 \pm 0.027$ & $0.868 \pm 0.018$ & $\mathbf{0 . 8 8 0} \pm \mathbf{0 . 0 1 7}$ \\
\hline
\end{tabular}

(b) rec vs. talk

\begin{tabular}{|c|c|c|c|c|c|c|}
\hline $\mathrm{m}$ & ASVM & CDSVM & MKL & SVMFR & DMKLDF & OKCDA \\
\hline 0 & & & $0.716 \pm 0.000$ & $\mathbf{0 . 8 0 1} \pm \mathbf{0 . 0 0 0}$ & $0.719 \pm 0.000$ & $0.702 \pm 0.000$ \\
\hline 1 & $0.737 \pm 0.097$ & $0.742 \pm 0.021$ & $0.742 \pm 0.022$ & $\mathbf{0 . 7 6 5} \pm \mathbf{0 . 0 4 3}$ & $0.755 \pm 0.022$ & $0.746 \pm 0.027$ \\
\hline 3 & $0.801 \pm 0.043$ & $0.780 \pm 0.020$ & $0.781 \pm 0.021$ & $0.776 \pm 0.094$ & $\mathbf{0 . 8 2 0} \pm \mathbf{0 . 0 1 7}$ & $0.786 \pm 0.023$ \\
\hline 5 & $0.815 \pm 0.047$ & $0.810 \pm 0.020$ & $0.809 \pm 0.021$ & $0.806 \pm 0.081$ & $\mathbf{0 . 8 2 2} \pm \mathbf{0 . 0 3 5}$ & $0.818 \pm 0.026$ \\
\hline 7 & $0.834 \pm 0.047$ & $0.832 \pm 0.015$ & $0.830 \pm 0.017$ & $0.824 \pm 0.077$ & $\mathbf{0 . 8 5 1} \pm \mathbf{0 . 0 3 1}$ & $0.841 \pm 0.022$ \\
\hline 10 & $0.857 \pm 0.011$ & $0.856 \pm 0.013$ & $0.855 \pm 0.013$ & $0.861 \pm 0.017$ & $0.881 \pm 0.027$ & $\mathbf{0 . 8 8 3} \pm \mathbf{0 . 0 2 1}$ \\
\hline
\end{tabular}

Table 3: Mean and Standard Deviation (in Percentages) of Classification Accuracies for All Methods with Different Number of Positive and Negative Training Samples from the Target Domain on the 20 Newsgroups dataset. The best results are marked with bold.

all methods is represented linearly ranged from 0 to 2 , while it becomes steady when $\mathrm{C}$ is greater than 2 . $\mathrm{C}=2$ seems to be a turning point. The result shows that the classification accuracy does not depend strongly on the value of regularization parameter $C$ and the existence of a stable value of $C$. It is interesting to note that our proposed method slightly underperforms others in terms of classification accuracy as $C$ ranged from 0.1 to 2 , but it outperforms the other methods in most cases when $C$ is greater than 2.

To take a deeper look at Table 3 , we analyse the performance of our method in comparison with other methods.

- In Fig 2(a) We observe that ASVM performs poorly for all cases. This may be attributed to the distribution difference between the source domain and the target domain. When $m$ is set to 0 , the performance of our method is not better than other methods, because the unlabeled patterns are not 


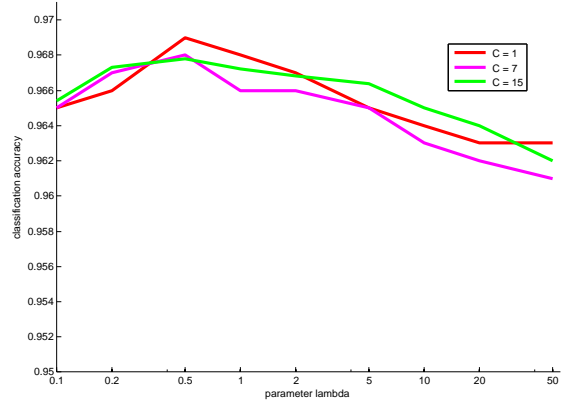

(a) User00 vs. User01

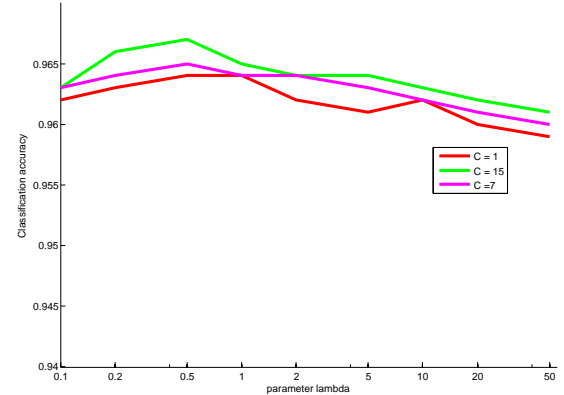

(b) User01 vs. User02

Figure 3: Performance (i.e, the means of classification accuracies) of OKCDA on the Email Spam dataset with different balance parameter $\lambda \in\{0.1,0.2,0.5,1,2,5,10,20,50\}$, and we set $m=15$.

utilized in this case. Note that when $m$ is greater than 0 , our proposed method is consistently better than all other methods in terms of mean classification accuracies. As $\mathrm{m}$ becomes larger, the advantage becomes more evident.

- In Fig 2(b), we observe that SVMFR have outperforms all other methods when $\mathrm{m}$ is set to 0 , which is unexpected. We can see DMKLDF have a stable performance in most cases. Our proposed method outperforms all other case when $\mathrm{m}$ is set to 10 . From Table 2(a) and Table 2(b), we have the following conclusions: many cross-domain learning methods generally achieve similar performances, and our proposed kernel choice method for cross-domain learning is better than most other methods in terms of the means of classification accuracies on datasets.

\subsection{Results of Email Spam Dataset}

For the Email Spam classification task, we also provide comparisons between OKCDA and other related methods. For each setting, we report the results of all methods by using the training data from the source domain as well as $m$ positive and $m$ negative training samples randomly selected from the target domain, where $m=0,1,3,7,10,15$ for the Email Spam. We randomly sample the 
training data from the target domain for five times. In Table 4 , we report the means and standard deviations of classification accuracies for all methods on the Email Spam datasets, respectively. Also noted that for all methods, each result in Table 4 is the best among all the result obtained by using different regularization parameters $C \in\{0.1,0.2,0.5,1,2,5,10,20,50\}$. From Table 4 , we have the following observations:

- The performance of these methods has not been changed greatly under the change of parameter $\mathrm{m}$. OKCDA and DMKLDF have the similar results and smallest standard deviation of both are significantly less than other methods. This fact demonstrates that OKCDA and DMKLDF have a relatively high stability.

- The experimental performance of our method is better than CDSVM, MKL and SVMFR. Compared with DMKLDF, our method has an approximate performance, which achieves a gap of 0.001 level.

- Since the change of $\mathrm{m}$ has not improved the performance of these methods, we believe that there exists a limit among these cross-domain learning methods. In other words, when the number of samples exceeds a certain threshold, further increasing the training set size does not improve the performance.

- Our proposed method OKCDA consistently performs better than some methods in terms of the means classification accuracies on Email Spam dataset, thanks to the explicit modeling of the data distribution mismatch, as well as the successful utilization of the unlabeled data. As shown in Table 4 when the number of labeled positive and negative training samples from the target domain increases, OKCDA has similar performance with DMKLDF but the performance does not improve.

We also compare our proposed method OKCDA with other cross-domain learning methods, including ASVM, CDSVM ,SVMFR and DMKLDF, by using different regularization parameter $C \in\{0.1,0.2,0.5,1,2,5,10,20,50\}$. The 
(a) User00 vs. Use01

\begin{tabular}{|c|c|c|c|c|c|c|}
\hline $\mathrm{m}$ & ASVM & CDSVM & MKL & SVMFR & DMKLDF & OKCDA \\
\hline 0 & & & $0.961 \pm 0.000$ & $0.957 \pm 0.000$ & $\mathbf{0 . 9 6 8} \pm \mathbf{0 . 0 0 0}$ & $0.963 \pm 0.000$ \\
\hline 1 & $0.959 \pm 0.006$ & $0.961 \pm 0.001$ & $0.962 \pm 0.022$ & $0.674 \pm 0.043$ & $\mathbf{0 . 9 6 8} \pm \mathbf{0 . 0 0 0}$ & $0.963 \pm 0.001$ \\
\hline 3 & $0.957 \pm 0.005$ & $0.962 \pm 0.001$ & $0.962 \pm 0.001$ & $0.948 \pm 0.010$ & $\mathbf{0 . 9 6 8} \pm \mathbf{0 . 0 0 1}$ & $0.964 \pm 0.002$ \\
\hline 7 & $0.959 \pm 0.005$ & $0.963 \pm 0.001$ & $0.963 \pm 0.001$ & $0.947 \pm 0.014$ & $\mathbf{0 . 9 6 8} \pm \mathbf{0 . 0 0 1}$ & $0.964 \pm 0.001$ \\
\hline 10 & $0.955 \pm 0.009$ & $0.964 \pm 0.000$ & $0.964 \pm 0.002$ & $0.946 \pm 0.019$ & $\mathbf{0 . 9 6 9} \pm \mathbf{0 . 0 0 1}$ & $0.965 \pm 0.001$ \\
\hline 15 al1 & $0.952 \pm 0.010$ & $0.966 \pm 0.020$ & $0.965 \pm 0.002$ & $0.947 \pm 0.010$ & $\mathbf{0 . 9 6 9} \pm \mathbf{0 . 0 0 1}$ & $0.965 \pm 0.001$ \\
\hline
\end{tabular}

(b) User01 vs. User02

\begin{tabular}{|c|c|c|c|c|c|c|}
\hline $\mathrm{m}$ & ASVM & CDSVM & MKL & SVMFR & DMKLDF & OKCDA \\
\hline 0 & & & $0.969 \pm 0.000$ & $0.668 \pm 0.000$ & $\mathbf{0 . 9 7 7} \pm \mathbf{0 . 0 0 0}$ & $0.972 \pm 0.000$ \\
\hline 1 & $0.964 \pm 0.006$ & $0.969 \pm 0.001$ & $0.970 \pm 0.022$ & $0.942 \pm 0.043$ & $\mathbf{0 . 9 7 7} \pm \mathbf{0 . 0 0 0}$ & $0.972 \pm 0.000$ \\
\hline 3 & $0.960 \pm 0.0018$ & $0.970 \pm 0.001$ & $0.970 \pm 0.021$ & $0.942 \pm 0.031$ & $\mathbf{0 . 9 7 7} \pm \mathbf{0 . 0 0 0}$ & $0.972 \pm 0.001$ \\
\hline 7 & $0.963 \pm 0.011$ & $0.970 \pm 0.001$ & $0.970 \pm 0.000$ & $0.949 \pm 0.018$ & $\mathbf{0 . 9 7 7} \pm \mathbf{0 . 0 0 0}$ & $0.972 \pm 0.001$ \\
\hline 10 & $0.967 \pm 0.005$ & $0.971 \pm 0.001$ & $0.970 \pm 0.001$ & $0.957 \pm 0.004$ & $\mathbf{0 . 9 7 7} \pm \mathbf{0 . 0 0 0}$ & $0.973 \pm 0.000$ \\
\hline 15 & $0.969 \pm 0.001$ & $0.972 \pm 0.001$ & $0.970 \pm 0.000$ & $0.961 \pm 0.002$ & $\mathbf{0 . 9 7 7} \pm \mathbf{0 . 0 0 2}$ & $0.973 \pm 0.001$ \\
\hline
\end{tabular}

Table 4: Mean and Standard Deviation (in Percentages) of Classification Accuracies for All Methods with Different Number of Positive and Negative Training Samples from the Target Domain on the Email Spam dataset. The best results are marked with bold.

result of all methods are also obtained by using $m$ positive and $m$ negative training sample from the target domain as well as the training samples from the source domain. We set $m=7$ and $m=15$ for Email Spam datasets in Fig. 2 . From the Fig. 2, we observe that when $C$ becomes larger, all methods tend to have better performances. However, OKCDA has relatively stable performance in terms of the standard deviations of classification accuracies.

4.5. An analysis of the $\lambda$ tradeoff between the structural risk and the bias minimization

In this subsection, we mainly investigate how would the choice of $\lambda$ affect the adaptation classification accuracy. Fig. 3 shows the variation in classification accuracies with varying $\lambda$ of $[0.1,0.2,0.5,1,2,5,10,20,50]$, where we set $\mathrm{m}=15$ and the regularization parameter $\mathrm{C}=1,7$ and 15 . Noted that the number of labeled samples from the two domains and the number of unlabeled samples from the target domain are almost the same on the Email Spam dataset. The 
x-axis in Fig. 3 are in logarithmic scale. We have the following observations:

- The performance of OKCDA changes with different $\lambda$ values. $\lambda$ is a hyperparameter that the model is not sensitive to, and needs to be tuned across different labeled or unlabeled data sizes.

- When $\lambda$ ranges from 0.1 to 50 , the classification accuracy changes with a small scale. When the $\lambda$ is gradually increasing, the performance decreases a bit.

- When $\lambda$ takes the value around 5 , the best performance of OKCDA can be obtained. In this case, both the labeled data and the unlabeled data from the target domain can be effectively utilized to learn a robust classifier.

\section{Conclusions}

In this paper, we propose a method of optimal kernel choice for domain adaption, namely, OKCDA. The paper extends the previous work on optimal kernel choice to two-sample tests, with an additional component to minimize the structural risk on the labeled data. The method is tested on the 20 Newsgroups dataset and Email Spam dataset, and compared to several existing methods. In our experiments, the kernel is set to be a linear combination of some base kernels. The kernel parameters are chosen to minimize the structural risk functional and the distribution bias between the samples from the auxiliary and target domains. To reduce the distribution mismatch, we minimize the Type II error based on the MMD and construct a test statistic between source domain and target domain. We will investigate how to choose the kernel automatically and explore the relationship between Type II error or Type I error and domain adaption learning performance in the future.

\section{Acknowledgments}

This work was supported in part by the National Natural Science Foundation of China under Grant 61370149, in part by the Fundamental Research Funds 
for the Central Universities (No. ZYGX2013J083), and in part by the Scien-

tific Research Foundation for the Returned Overseas Chinese Scholars, State Education Ministry.

\section{References}

[1] S. Zhong, X. Zeng, S. Wu, L. Han, Sensitivity-based adaptive learning rules for binary feedforward neural networks, IEEE Trans. Neural Networks and Learning Systems. 23 (3) (2012) 480-491.

[2] S. J. Pan, I. W. Tsang, J. T. Kwok, Q. Yang, Domain adaptation via transfer component analysis, IEEE Trans. Neural Networks. 22 (2) (2011) $199-210$.

[3] Z. Zhang, J. Zhou, Multi-task clustering via domain adaptation, Pattern Recognition. 45 (1) (2012) 465-473.

[4] S. J. Pan, J. T. Kwok, Q. Yang, Transfer learning via dimensionality reduction, in: Association for the Advancement of Artificial Intelligence, Vol. 8, 2008, pp. 677-682.

[5] X. Wu, D. Xu, L. Duan, J. Luo, Action recognition using context and appearance distribution features, in: IEEE 2011 Conference on Computer Vision and Pattern Recognition, 2011, pp. 489-496.

[6] J. Blitzer, M. Dredze, F. Pereira, Biographies, bollywood, boom-boxes and blenders: Domain adaptation for sentiment classification, in: Annual Meeting of the Association for Computational Linguistics, Vol. 7, 2007, pp. 440447.

[7] L. Duan, D. Xu, I. H. Tsang, J. Luo, Visual event recognition in videos by learning from web data, IEEE Trans. Pattern Analysis and Machine Intelligence. 34 (9) (2012) 1667-1680. 
[8] D. Xu, S. F. Chang, Video event recognition using kernel methods with

[15] L. Dong, E. Izquierdo, A topology synthesizing approach for classification of visual information, in: CBMI 2008 International Workshop on ContentBased Multimedia Indexing, 2008, pp. 373-380.

[16] H. Daume III, Frustratingly easy domain adaptation, in: Proceedings of 375 multilevel temporal alignment, IEEE Trans. Pattern Analysis and Machine Intelligence. 30 (11) (2008) 1985-1997.

[9] D. Vzquez, A. M. Lpez, D. Ponsas, Virtual worlds and active learning for human detection, in: Proceedings of the 13th international conference on multimodal interfaces, 2011, pp. 393-400.

[10] D. Vzquez, A. Lpez, D. Ponsa, J. Marin, Cool world: domain adaptation of virtual and real worlds for human detection using active learning, in: Advances in Neural Information Processing Systems-Workshop on Domain, 2014.

[11] Y.-G. Jiang, J. Wang, S.-F. Chang, C. W. Ngo, Domain adaptive semantic diffusion for large scale context-based video annotation, in: IEEE 12th International Conference on Computer Vision, 2009, pp. 1420-1427.

[12] J. Yang, R. Yan, A. G. Hauptmann, Cross-domain video concept detection using adaptive svms, in: Proceedings of the 15th international conference on Multimedia, 2007, pp. 188-197.

[13] W. Jiang, E. Zavesky, S. F. Chang, A. Loui, Cross-domain learning methods for high-level visual concept classification, in: IEEE 15th International Conference on Image Processing, 2008, pp. 161-164.

[14] L. Dong, J. Su, E. Izquierdo, Scene-oriented hierarchical classification of blurry and noisy images, IEEE Trans. Image Processing. 21 (5) (2012) $2534-2545$.

the 45th Annual Meeting of the Association of Computational Linguistics, 2007, pp. 256-263. 
[17] L. Duan, I. W. Tsang, D. Xu, Domain transfer multiple kernel learning, IEEE Trans. Pattern Analysis and Machine Intelligence. 34 (3) (2012) 465479 .

[18] G. R. Lanckriet, N. Cristianini, P. Bartlett, L. E. Ghaoui, M. I. Jordan, Learning the kernel matrix with semidefinite programming, The Journal of Machine Learning Research. 5 (2004) 27-72.

[19] L. Duan, D. Xu, I. W. Tsang, Domain adaptation from multiple sources: A domain-dependent regularization approach, IEEE Trans. Neural Networks and Learning Systems. 23 (3) (2012) 504-518.

[20] Y. Lu, L. Wang, J. Lu, J. Yang, C. Shen, Multiple kernel clustering based on centered kernel alignment, Pattern Recognition. 47 (11) (2014) 36563664 .

[21] A. Salah, H. M. Mohammand, Z. Xiao, M. V. Richard, Human activity recognition using multi-features and multiple kernel learning, Pattern Recognition. 47 (5) (2014) 1800-1812.

[22] M. Varma, B. R. Babu, More generality in efficient multiple kernel learning, in: Proceedings of the 26th Annual International Conference on Machine Learning, 2009, pp. 1065-1072.

[23] J. Bootkrajang, A. Kabán, Learning kernel logistic regression in the presence of class label noise, Pattern Recognition. 47 (11) (2014) 3641-3655.

[24] H. Jia, Y. M. Cheung, J. Liu, Cooperative and penalized competitive learning with application to kernel-based clustering, Pattern Recognition. 47 (9) (2014) 3060-3069.

400

[25] Z. Rached, F. Alajaji, L. Campbell, The kullback-leibler divergence rate between markov sources, IEEE Trans. on Information Theory. 50 (5) (2004) 917-921. 
[26] K. M. Borgwardt, A. Gretton, M. J. Rasch, H. P. Kriegel, B. Schölkopf, A. J. Smola, Integrating structured biological data by kernel maximum mean discrepancy, Bioinformatics. 22 (14) (2006) e49-e57.

[27] R. Rosipal, L. J. Trejo, Kernel partial least squares regression in reproducing kernel hilbert space, Journal of Machine Learning Research. 2 (2) (2002) 97-123.

[28] J. Huang, A. Gretton, K. M. Borgwardt, B. Schölkopf, A. J. Smola, Correcting sample selection bias by unlabeled data, in: Advances in neural information processing systems, 2006, pp. 601-608.

[29] A. Gretton, D. Sejdinovic, H. Strathmann, S. Balakrishnan, M. Pontil, K. Fukumizu, B. K. Sriperumbudur, Optimal kernel choice for large-scale two-sample tests, in: Advances in Neural Information Processing Systems, 2012, pp. 1205-1213.

[30] G. Schweikert, G. Rätsch, C. Widmer, B. Schölkopf, An empirical analysis of domain adaptation algorithms for genomic sequence analysis, in: Advances in Neural Information Processing Systems, 2009, pp. 1433-1440.

[31] U. D. Vzquez, A. Lpez, D. Ponsa, Unsupervised domain adaptation of virtual and real worlds for pedestrian detection, in: International Conference on Pattern Recognition, 2012, pp. 3492-3495.

[32] J. Xu, S. Ramos, D. Vzquez, A. Lpez, Domain adaptation of deformable part-based models, IEEE Trans. Pattern Analysis and Machine Intelligence. 36 (12) (2014) 2367-2380.

[33] M. Varma, D. Ray, Learning the discriminative power-invariance trade-off, in: IEEE 11th International Conference on Computer Vision, 2007, pp. $1-8$.

[34] A. Yanagawa, S. F. Chang, L. Kennedy, W. Hsu, Columbia university baseline detectors for 374 lscom semantic visual concepts, Columbia University ADVENT technical report. (2007) 222-2006. 